\title{
RECURRENT AND PERIODIC POINTS IN DENDRITIC JULIA SETS
}

\author{
ALEXANDER BLOKH \\ (Communicated by Bryna Kra) \\ Dedicated to A. N. Sharkovskiy on the occasion of his 75th birthday \\ Abstract. We relate periodic and recurrent points in dendritic Julia sets. \\ This generalizes well-known results for interval dynamics.
}

\section{INTRODUCTION AND THE MAIN RESULTS}

There are two types of results in continuous interval dynamics. First, there are the results dealing with periods of periodic points. The main one here is an amazing fact established by A. N. Sharkovskiy at the beginning of the 1960s in Sha64 and describing the coexistence among periods of periodic points of an interval map. To state it we need the following definitions (in what follows we assume the knowledge of a few basic notions such as periodic point, cycle, etc.; for the sake of completeness we define all other notions).

Definition 1.1 (Sharkovskiy ordering). Define the Sharkovskiy ordering for the set $\mathbb{N}$ of positive integers united with the symbol $2^{\infty}$ as follows:

$$
3 \succ 5 \succ \cdots \succ 2 \cdot 3 \succ 2 \cdot 5 \succ \cdots \succ 2^{\infty} \succ \cdots \succ 4 \succ 2 \succ 1 .
$$

Denote by $\operatorname{Sh}(k)$ the set of all positive integers $m$ such that $k \succ m$, together with $k$ (except when $k=2^{\infty}$, in which case the symbol $2^{\infty}$ is not included in $\operatorname{Sh}(k)$ ). Also, given a map $f$ we denote by $\operatorname{Per}(f)$ the set of the periods of all cycles of $f$ (by the period we mean the least period).

Now we are ready to state the celebrated Sharkovskiy Theorem [Sha64].

Theorem 1.2. If $f:[0,1] \rightarrow[0,1]$ is a continuous map, $m \succ n$ and $m \in \operatorname{Per}(f)$, then $n \in \operatorname{Per}(f)$. Therefore there exists $k \in \mathbb{N} \cup\left\{2^{\infty}\right\}$ such that $\operatorname{Per}(f)=\operatorname{Sh}(k)$. Conversely, if $k \in \mathbb{N} \cup\left\{2^{\infty}\right\}$, then there exists a continuous interval map $f$ such that $\operatorname{Per}(f)=\operatorname{Sh}(k)$.

One can safely say that Theorem 1.2 started combinatorial one-dimensional $d y$ namics. Papers in this field either seek to specify the coexistence of periods of cycles for interval maps (e.g., such papers on the so-called "rotation theory for interval maps"; see [Blo95a, BM97]) or attempt to extend a version of the result onto other

\footnotetext{
Received by the editors August 26, 2011 and, in revised form, December 16, 2011 and January 5, 2012 .

2010 Mathematics Subject Classification. Primary 37B45; Secondary 37C25, 37E05, 37E25, 37F10, 37F50.

Key words and phrases. Periodic points, recurrent points, Julia set.

The author was partially supported by NSF grant DMS-0901038.
} 
one-dimensional maps, such as maps of "graphs", i.e., of compact one-dimensional branched manifolds (see ALM00, where the main topics in one-dimensional dynamics are nicely covered and an extensive list of references is provided).

Results of the second type deal with all limit sets rather than only periodic orbits. This direction has also been initiated by Sharkovskiy, who studied maps of the interval from this perspective as well. Still, these developments seem to be less well-known. To state one of Sharkovskiy's results in this area (the one which we will generalize in this paper), we need the following definition.

Definition 1.3 (Limit sets and recurrent points). Suppose that $g: X \rightarrow X$ is a continuous map of a compact metric space $X$ to itself. Given a point $x \in X$, the sequence of points $x, g(x), \ldots$ is called the orbit of $x$. The set $\omega(x)$ of all limit points of the orbit of $x$ is said to be the limit set of $x$; a point of a limit set is often called simply a limit point. A point which belongs to its own limit set is said to be recurrent.

The next definition is a little less standard.

Definition 1.4 (Center of a dynamical system). Suppose that $g: X \rightarrow X$ is a continuous map of a compact metric space $X$. The center of a dynamical system $g$ is the closure of the set of all its recurrent points. Equivalently, the center of $g$ can be defined as the smallest invariant closed set $C_{g}$ such that for any invariant probability measure $\mu$ we have that $\mu\left(C_{g}\right)=1$.

The most obvious example of a recurrent point is a periodic point; in this case the recurrence manifests itself in the most transparent way. Thus, the center of a map must contain the closure of all periodic points of the map. On the other hand, the opposite inclusion fails already in the case of irrational circle rotations. Thus, it is natural to ask in various cases how the set of all periodic points is related to the set of recurrent points (and thus to the center of a dynamical system). More generally, one can ask how the limit sets of all points are related to the set of periodic points of a map.

These problems were considered by A. N. Sharkovskiy in the $1960 \mathrm{~s}$, when a variety of results were obtained (see, e.g., [Sha64a, Sha66, Sha66a, Sha67, Sha68]). The scope of this paper does not allow us to go into a detailed description of this series of papers which, in our view, laid the foundation of the one-dimensional topological dynamics. Rather, we concentrate on the problems described in the previous paragraph and the way they were addressed in [Sha64a], where the following theorem was proven.

Theorem 1.5. The center of an interval map coincides with the closure of the set of all periodic points of the map.

One way of further developing one-dimensional topological dynamics is to show that results which are somewhat stronger than Sharkovskiy's results can be obtained if the maps are taken from a more narrow class than the class of continuous interval maps. If one stays within the framework of continuous interval maps (i.e., considers neither discontinuous nor smooth interval maps), then the most natural such class seems to be that of piecewise-monotone continuous interval maps. By piecewisemonotone continuous interval maps we mean continuous interval maps for which the interval can be partitioned, by finitely many points, into finitely many adjacent 
intervals on each of which the map is (non-strictly) monotone. The following result is due to Z. Nitecki Nit80.

Theorem 1.6. Suppose that $f$ is a piecewise-monotone continuous interval map. Then the limit set $\omega(x)$ of any point is contained in the closure of the set of all periodic points of $f$.

Theorems 1.5 and 1.6 are clearly related: Theorem 1.6 implies Theorem 1.5 in the piecewise-monotone case, yet Theorem 1.5 holds for all continuous interval maps. Examples constructed by Sharkovskiy show that Theorem 1.6 does not hold for all continuous interval maps. Also, we would like to mention here that in the above statements of Theorems 1.5] and 1.6 we omitted parts of the original formulations which are not directly related to the present work.

In this paper we extend Theorem [1.6 and Theorem 1.5] onto continuous maps of more complicated topological spaces. As mentioned above, there are quite a few papers in which dynamics was extended from the interval onto more complicated but still one-dimensional topological spaces within both one-dimensional combinatorial dynamics and one-dimensional topological dynamics; for the most part this was done for continuous maps of "graphs" (see, e.g., ALM00, Blo80s]).

However, here we generalize Theorems 1.5] and 1.6 onto one-dimensional spaces more complicated than "graphs". So far few results similar to those from onedimensional dynamics have been obtained for such topological spaces (see, e.g., [MT89, AEO07]). First we need the following definition.

Definition 1.7 (Dendrites, their points and subarcs). A dendrite is a non-degenerated locally connected continuum which does not contain Jordan curves. A point $x$ of a dendrite $X$ is called an endpoint of $X$ if $X \backslash\{x\}$ is connected, a cutpoint of $X$ if $X \backslash\{x\}$ is disconnected and a branchpoint of $X$ if $X \backslash\{x\}$ has more than two components. For any two points $a, b \in X$ there exists a unique closed $\operatorname{arc}$ in $X$ with endpoints $a$ and $b$ denoted $[a, b]$; the notation $(a, b),(a, b]$ and $[a, b)$ is analogous to similar notation in the interval case.

As dendrites are much more complicated topological spaces than the interval or even a tree (i.e., a dendrite with finitely many branchpoints), it is natural to adjust some of the definitions for them so that tools of one-dimensional dynamics will apply.

Definition 1.8. Let $f: X \rightarrow X$ be a continuous self-mapping of a dendrite $X$. Suppose that for points $x, b \in X$ there exist an arc $[a, b] \subset X$ and a sequence of images $f^{n_{k}}(x) \in(a, b)$ of $x$ which converge to $b$. Then we say that $b$ is a limit point of $x$ of arc type; denote the set of all limit points of $x$ of arc type by $\omega^{a}(x)$. If $b$ is a limit point of $b$ of arc type, then we say that $b$ is a recurrent point of arc type.

If $y$ is a limit point of $x$ which is not of arc type, then we call $y$ a limit point of $x$ of non-separating type; denote the set of all limit points of $x$ of non-separating type by $\omega^{n s}(x)$. If $y$ is a limit point of $y$ of non-separating type, we call $y$ a recurrent point of non-separating type.

By definition, $\omega^{a}(x) \cup \omega^{n s}(x)=\omega(x)$. Also, observe that if $b$ is a limit point of $x$ of arc type, then infinitely many points $f^{n_{k}}(x)$ are cutpoints of $X$. In the case of limit points of arc type the convergence resembles that which takes place in the interval case. It is then no wonder that limit points of arc type and recurrent points 
of arc type play a role for dendrites similar to that of limit points and recurrent points on the interval.

Theorem 1.9. Let $f: X \rightarrow X$ be a continuous self-mapping of a dendrite $X$. Then all recurrent points of arc type belong to the closure of the set of all periodic points.

Clearly, Theorem 1.9 implies Theorem 1.5 in the case when $X$ is a finite tree. Indeed, if $X$ is a finite tree, then all its recurrent non-periodic points are of arc type. Hence in that case all recurrent points belong to the closure of the set of all periodic points. Since the center of $f$ is the closure of all its recurrent points, Theorem 1.5 follows. Thus, Theorem 1.9 can be viewed as a generalization of Theorem 1.5 for dendrites. The corresponding generalization of Theorem 1.6 requires considering a more narrow class of maps of dendrites; on the other hand, the results in that case are more precise, as we now consider periodic cutpoints rather than just periodic points. As such a class, we choose topological polynomials on their dendritic Julia sets. Thus, our research is triggered not only by the desire to further study onedimensional dynamics, but also by the interest to complex, in particular polynomial, dynamics (so that the obtained results can be considered as a part of both onedimensional and complex dynamics).

Indeed, it is well-known that a polynomial $P$ on its locally connected Julia set can be described using the appropriate lamination $\sim$, i.e., specific equivalence relation $\sim$ on the circle $\mathbb{S}^{1}$ (notice that $\sim$-classes in this case are always finite). The corresponding quotient space $J_{\sim}$ of $\mathbb{S}^{1}$ is then called a topological Julia set, while the map $f_{\sim}: J_{\sim} \rightarrow J_{\sim}$ induced by $\sigma_{d}=z^{d}: \mathbb{S}^{1} \rightarrow \mathbb{S}^{1}$ (here $d \geq 2$ is the degree of $P$ ) is called a topological polynomial. Since the original Julia set $J_{P}$ of $P$ is assumed here to be locally connected, it follows that $\left.P\right|_{J_{P}}$ and $\left.f_{\sim}\right|_{J_{\sim}}$ are topologically conjugate.

Even though there are, of course, locally connected Julia sets which are not dendrites, results on the dendritic case as a rule contain the most substantial steps of the proofs; then these proofs can often be extended onto all Julia sets modulo overcoming technical difficulties. Therefore we believe that studying dendritic Julia sets is a proper way of developing topological dynamics of polynomials on their locally connected Julia sets (in fact, later on we plan to extend our results onto the general case of locally connected Julia sets). We need the following definition.

Definition 1.10 (Persistent cutpoints). A point $x$ is a persistent cutpoint of $J_{\sim}$ if all its images are cutpoints of $J_{\sim}$.

This is not restrictive, as the only cutpoints which are not persistent are preimages of (some) critical points of $f_{\sim}$; in what follows we talk about limit points of persistent cutpoints rather than all cutpoints. Observe also that in Theorem 1.11 we talk about the entire limit set of $x$ and not only the set $\omega^{a}(x)$ of all limit points of $x$ of arc type.

Theorem 1.11. Let $f_{\sim}$ be a topological polynomial with dendritic Julia set $J_{\sim}$, let $X \subset J_{\sim}$ be an invariant dendrite and let $x \in X$ be a persistent cutpoint of $X$. Then $\omega(x)$ is contained in the closure of the set of all periodic cutpoints of $\left.f_{\sim}\right|_{X}$. In particular, the limit set of any persistent cutpoint $x$ of $J_{\sim}$ is contained in the closure $\overline{\mathrm{PC}_{\sim}}$ of the set $\mathrm{PC}_{\sim}$ of all periodic cutpoints of $f_{\sim}$, and all recurrent persistent cutpoints belong to $\overline{\mathrm{PC}_{\sim}}$.

It is easy to see that Theorem 1.11 implies Theorem 1.9 for topological polynomials with dendritic Julia sets. Indeed, a limit point of arc type (in particular, a 
recurrent point of arc type) belongs to the appropriate limit set which is the limit set of a persistent cutpoint. Then by Theorem 1.11 a limit (recurrent) point of arc type belongs to the closure of all periodic cutpoints. This statement is even stronger than that of Theorem 1.9.

Theorem 1.11 allows us to make conclusions about invariant measures of $f_{\sim}$. Namely, we prove Corollary 1.12 in it, when we say that a probability measure $\mu$ is supported on a set $A$, we mean that $\mu(A)=1$.

Corollary 1.12. Suppose that $\mu$ is a probability invariant measure of $f_{\sim}$. Then it can be represented as the convex sum of two probability invariant measures $\mu_{e}$ (supported on the set of all endpoints of $J_{\sim}$ ) and $\mu_{c}$ (supported on the set of cutpoints of $J_{\sim}$ intersected with the closure of the set of all periodic cutpoints).

\section{Preliminaries}

In this section we introduce the notions necessary to obtain the announced results. We also state some useful lemmas.

2.1. Laminations. We begin with laminations introduced by Thurston in [Thu85]. Laminations provide a combinatorial tool which allows us to deal with polynomial dynamics. We define laminations below; however, our approach is somewhat different from Thu85] (cf. BL02a]).

Definition 2.1 (Laminations as equivalence relations). An equivalence relation $\sim$ on the unit circle $\mathbb{S}^{1}$ is called a lamination if it has the following properties:

(E1) the graph of $\sim$ is a closed subset in $\mathbb{S}^{1} \times \mathbb{S}^{1}$;

(E2) if $t_{1} \sim t_{2} \in \mathbb{S}^{1}$ and $t_{3} \sim t_{4} \in \mathbb{S}^{1}$, but $t_{2} \not t_{3}$, then the open straight line segments in $\mathbb{C}$ with endpoints $t_{1}, t_{2}$ and $t_{3}, t_{4}$ are disjoint;

(E3) each equivalence class of $\sim$ is totally disconnected.

Consider the map $\sigma_{d}: \mathbb{S}^{1} \rightarrow \mathbb{S}^{1}$ defined by the formula $\sigma_{d}(z)=z^{d}(d \geq 2)$.

Definition 2.2 (Dynamics and invariant laminations). A lamination $\sim$ is called $\left(\sigma_{d^{-}}\right)$invariant if:

(D1) is forward invariant: for a class $g$, the set $\sigma_{d}(g)$ is a class too;

(D2) $\sim$ is backward invariant: for a class $g$, its preimage $\sigma_{d}^{-1}(g)=\left\{x \in \mathbb{S}^{1}\right.$ : $\left.\sigma_{d}(x) \in g\right\}$ splits into at most $d$ classes;

(D3) for any $\sim$-class $g$, the map $\sigma_{d}: g \rightarrow \sigma_{d}(g)$ extends to $\mathbb{S}^{1}$ as an orientation preserving covering map such that $g$ is the full preimage of $\sigma_{d}(g)$ under this covering map;

(D4) all $\sim$-classes are finite.

Part (D3) of Definition 2.1 has an equivalent version. A (positively oriented) hole $(a, b)$ of a compactum $Q \subset \mathbb{S}^{1}$ is a component of $\mathbb{S}^{1} \backslash Q$ such that moving from $a$ to $b$ inside $(a, b)$ is in the positive direction. Then (D3) is equivalent to the fact that for a $\sim$-class $g$ either $\sigma_{d}(g)$ is a point or for each positively oriented hole $(a, b)$ of $g$ the positively oriented arc, $\left(\sigma_{d}(a), \sigma_{d}(b)\right)$ is a positively oriented hole of $\sigma_{d}(g)$.

For a $\sigma_{d}$-invariant lamination $\sim$ we consider the topological Julia set $\mathbb{S}^{1} / \sim=J_{\sim}$ and the topological polynomial $f_{\sim}: J_{\sim} \rightarrow J_{\sim}$ induced by $\sigma_{d}$. The quotient map $p_{\sim}: \mathbb{S}^{1} \rightarrow J_{\sim}$ extends to the plane with the only non-trivial fibers being the convex hulls of $\sim$-classes. Using Moore's Theorem one can extend $f_{\sim}$ to a branchedcovering map $f_{\sim}: \mathbb{C} \rightarrow \mathbb{C}$ of the same degree. The complement of the unbounded 
component of $\mathbb{C} \backslash J_{\sim}$ is called the filled-in topological Julia set and is denoted $K_{\sim}$. If the lamination $\sim$ is fixed, we may omit $\sim$ from the notation.

For points $a, b \in \mathbb{S}^{1}$, let $\overline{a b}$ be the chord with endpoints $a$ and $b$ (if $a=b$, set $\overline{a b}=\{a\})$. For $A \subset \mathbb{S}^{1}$ let $\mathrm{Ch}(A)$ be the convex hull of $A$ in $\mathbb{C}$.

Definition 2.3 (Geometric laminations, their leaves and gaps). If $A$ is a $\sim$-class, we call an edge $\overline{a b}$ of $\operatorname{Bd}(\mathrm{Ch}(A))$ a leaf. The family of all leaves of $\sim$, denoted by $\mathcal{L}_{\sim}$, is called the geometric lamination generated by $\sim$. Denote the union of all leaves of $\mathcal{L}_{\sim}$ by $\mathcal{L}_{\sim}^{+}$. Extend $\sigma_{d}$ (keeping the notation) linearly over all individual chords in $\overline{\mathbb{D}}$, in particular over leaves of $\mathcal{L}_{\sim}$. Note that even though the extended $\sigma_{d}$ is not well defined on the entire disk, it is well defined on every individual chord in the disk.

The two-point $\sim$-class (and its convex hull) is said to be a leaf-class. The closure of a non-empty component of $\mathbb{D} \backslash \mathcal{L}_{\sim}^{+}$is called a gap of $\sim$. If $G$ is a gap, we talk about edges of $G$; thus a leaf is either a leaf-class or an edge of a gap. If $G$ is a gap or leaf, we call the set $G^{\prime}=\mathbb{S}^{1} \cap G$ the basis of $G$. A degenerate -class is said to be a bud of $\sim$. In what follows for $y \in J_{\sim}$ we denote by $G_{y}=\mathrm{Ch}\left(p_{\sim}^{-1}(y)\right)$ the gap, leaf-class or bud corresponding to $y$ under $p_{\sim}$.

A gap or leaf $U$ is said to be preperiodic if for some minimal $m$ the set $\sigma_{d}^{m}(U)$ is periodic, $m>0$, and $U, \ldots, \sigma_{d}^{m-1}(U)$ are not periodic. Then the number $m$ is called the preperiod of $U$. If $U$ is either periodic or preperiodic, we will call it (pre)periodic. Similarly we treat critical, precritical and (pre)critical objects.

2.2. Existence of fixed cutpoints. In this subsection we state the results of BFMOT10 concerning the existence of fixed cutpoints in non-invariant continua (in particular, non-invariant subcontinua of $J_{\sim}$ ). The main results of BFMOT10. are much more general; however, we need only those which apply to topological polynomials with dendritic Julia sets. We will show how to modify some of the results of BFMOT10 to our needs. However first we need a few definitions introduced in BFMOT10.

Definition 2.4 (Boundary scrambling for dendrites). Suppose that $f$ maps a dendrite $D_{1}$ to a dendrite $D_{2} \supset D_{1}$. Put $E=\overline{D_{2} \backslash D_{1}} \cap D_{1}$ (observe that $E$ may be infinite). If for each non-fixed point $e \in E, f(e)$ is contained in a component of $D_{2} \backslash\{e\}$ which intersects $D_{1}$, then we say that $f$ has the boundary scrambling property or that it scrambles the boundary. Observe that if $D_{1}$ is invariant, then $f$ automatically scrambles the boundary.

Now we can state a combined and simplified version of Lemma 7.2.2(2) and Lemma 7.2.5 of [BFMOT10].

Lemma 2.5. The following facts hold:

(1) Suppose that $f$ maps a dendrite $D_{1}$ to a dendrite $D_{2} \supset D_{1}$. Put $E=$ $\overline{D_{2} \backslash D_{1}} \cap D_{1}$. Moreover, suppose that $f$ scrambles the boundary. Then $f$ has a fixed point $a \in D_{1}$.

(2) If in the above situation there are no fixed points in $E, f=f_{\sim}$ is a topological polynomial, and $D_{2} \subset J_{\sim}$ is a subcontinuum of a dendritic topological Julia set, then a can be chosen to be a cutpoint of $D_{1}$.

Yet another result from BFMOT10 is Lemma 7.2.2(1), which is stated below. When talking about points in a dendrite $D$, we say that a point $x$ separates a point $y$ from a point $z$ if $y$ and $z$ belong to distinct components of $D \backslash\{x\}$. 
Lemma 2.6. Suppose that $f: X \rightarrow X$ is a continuous self-mapping of a dendrite $X$. Suppose that $a \neq b$ are points in $X$ such that $f(a)$ is separated from $b$ by $a$ and $f(b)$ is separated from $a$ by $b$. Then there exists a fixed point in $(a, b)$.

Finally, we state a result which immediately follows from Theorem 7.2.6 of BOPT11 and well-known properties of periodic points of topological Julia sets. Speaking of periodic cutpoints of a map $g: Y \rightarrow Y$, we mean cutpoints of $Y$ which are periodic (thus, if $Y \subset Z$, then we do not consider cutpoints of $Z$ which are endpoints of $Y$ as periodic cutpoints of $f: Y \rightarrow Y$ ).

Theorem 2.7. Suppose that $X \subset J_{\sim}$ is an invariant subdendrite of a topological Julia set $J_{\sim}$. Then there are infinitely many periodic cutpoints of $\left.f_{\sim}\right|_{X}$.

2.3. Dynamical core of topological polynomials. There are a few new results which to an extent relate the set of periodic cutpoints of $f_{\sim}$ to the set of limit points of persistent cutpoints as well as limit sets of some critical points. These results were recently obtained in BOPT11, Section 3 . In the case when $J_{\sim}$ is a dendrite, the main result (Theorem 3.12) of Section 3 of [BOPT11] can be stated as follows.

Theorem 2.8. In the case of a topological polynomial $f_{\sim}$ with dendritic Julia set $J_{\sim}$, the minimal invariant continuum containing limit sets of all persistent cutpoints of $f_{\sim}$ and the minimal invariant continuum containing all periodic cutpoints of $f_{\sim}$ coincide. Moreover, this continuum (denote it $\mathrm{COR}_{f_{\sim}}$ ) coincides with the smallest invariant continuum containing all critical points of $f_{\sim}$ which belong to $\mathrm{COR}_{f_{\sim}}$.

The continuum defined in Theorem 2.8 is called the dynamical core of $f_{\sim}$. Clearly, Theorem 2.8 relates the sets of points which we want to study. However this connection is not sufficiently precise, as in Theorem 2.8 we deal with minimal continua containing certain sets of points (such as the union of all limit points of persistent cutpoints and the set of all periodic cutpoints) rather than with these sets themselves. The present paper seeks to improve and specify these results by establishing, at least in the case of dendrites, the connection between the sets themselves.

We will need the following lemma, which is a simplified version of Lemma 3.11 of [BOPT11] as it applies in the case when $J_{\sim}$ is a dendrite.

Lemma 2.9. Suppose that $X \subset J_{\sim}$ is an invariant continuum and $x \in X$ is a cutpoint of $X$. Then there exists $n$ such that $f_{\sim}^{n}(x)$ belongs to the minimal invariant continuum containing all critical points of $f_{\sim}$ which belong to $X$.

\section{MAin RESUlts}

For brevity in what follows we will often omit $\sim$ from the notation (thus, we write $J$ instead of $J_{\sim}, f$ instead of $f_{\sim}$, etc.). Also, we often write $\sigma$ instead of $\sigma_{d}$.

We begin by considering the case of an endpoint. It turns out to be easier; still it shows the way our tools apply.

Lemma 3.1. Let $f: X \rightarrow X$ be a continuous self-mapping of a dendrite $X$ and let $b$ be an endpoint of $X$. Suppose that $b$ is a limit point of $x$ of arc type so that there exists an arc $[a, b] \subset X$ and a sequence of images $f^{n_{k}}(x) \in(a, b)$ of $x$ which converge to $b$. Then $b$ is a limit point of periodic points of $f$. If $X \subset J$ is an invariant subcontinuum of a topological dendritic Julia set $J$ and $f$ is a topological polynomial, then $b$ is in fact a limit point of periodic cutpoints of $\left.f\right|_{X}$. 
Proof. Fix some $k$. Let $V$ be the component of $X \backslash\left\{f^{n_{k}}(x)\right\}$ which contains $b$. We apply Lemma 2.5 to $\bar{V}$ and to the map $f^{n_{k+1}-n_{k}}$. Then by Lemma 2.5 (1) $\bar{V}$ contains a periodic point $z$ (actually, a $f^{n_{k+1}-n_{k}}$-fixed point $z$ ); moreover, since $f^{n_{k}}(x)$ is not $f^{n_{k+1}-n_{k}}$-fixed, then $z \in V$. Since $X$ is locally connected, this implies the lemma in the general case. On the other hand, if $f$ is a topological polynomial with dendritic Julia set $J$ and $X \subset J$, then by Lemma 2.5) (2) $b$ is a limit point of periodic cutpoints of $f$ as desired.

Clearly, this lemma proves Theorem 1.9 in the case when $b$ is an endpoint of $X$. It also proves in part Theorem 1.11 by showing that, in the case of a topological dendritic Julia set $J$ and a topological polynomial, an endpoint $b$ of $X \subset J$ which is a limit point of arc type is a limit point of periodic cutpoints. To deal with the general case we need the following result; it has a technical nature but implies many useful conclusions.

Lemma 3.2. Let $f: X \rightarrow X$ be a continuous self-mapping of a dendrite $X$. Suppose that $b$ is a limit point of $x$ of arc type so that there exists an arc $[a, b] \subset X$ and a sequence of images $f^{n_{k}}(x) \in(a, b)$ of $x$ which converge to $b$. Moreover, suppose that there exists $d \in(a, b)$ such that the component $B$ of $X \backslash\{d, b\}$, containing $(d, b)$, has the following properties:

(1) if we make no extra assumptions about $f$ and $X$, then we assume that $B$ does not contain any periodic points;

(2) if we are given that $X \subset J_{\sim}$ is a subset of a dendritic Julia set and $f_{\sim}$ is a topological polynomial, then we assume only that $B$ does not contain periodic cutpoints of $\left.f\right|_{X}$.

Then $b$ never enters $(d, b)$, and for every point $y \in(d, b)$ and any number $m$ such that $f^{m}(y) \in(d, b)$, we have that $f^{m}(y) \in(y, b)$. In particular, we may assume that $x \in(a, b), f^{n_{1}}(x), \ldots, f^{n_{k}}(x), \ldots$ are all images of $x$ which enter $(x, b)$ and that these points approach $b$ in a monotone fashion.

Proof. Let us introduce the following order among points of $[a, b]: z<y$ means that $y \in(z, b]$. We may assume that $d<x<f^{k}(x)$ for some $k$. Let us show then that $f^{2 k}(x)$ is contained in the component of $X \backslash\left\{f^{k}(x)\right\}$ which contains $b$. Indeed, suppose otherwise. Then there are two cases. First, it may happen that $f^{2 k}(x)$ is located in a component $V$ of $X \backslash\left\{f^{k}(x)\right\}$ which contains neither $x$ nor $b$. Then this component $V$ is inside $B$, and it follows by Lemma 2.5, applied to $V$ and $f^{k}$, that there is a periodic (actually, $f^{k}$-fixed) point (in case (2) of the lemma, cutpoint) inside $B$, a contradiction. Second, $f^{k}(x)$ may be contained in the component of $X \backslash\left\{f^{k}(x)\right\}$ containing $x$. Then we can consider $V$ as the component of $X \backslash\left\{x, f^{k}(x)\right\}$ containing $\left(x, f^{k}(x)\right)$. Again by Lemma 2.5 this implies that there is a periodic (actually, $f^{k}$-fixed) point (in case (2) of the lemma, cutpoint) inside $B$, a contradiction. Hence $f^{2 k}(x)$ belongs to the component of $X \backslash\left\{f^{k}(x)\right\}$ which contains $b$.

The arguments can be continued by induction. Indeed, assume that $f^{n k}(x)$ is contained in the component of $X \backslash\left\{f^{k}(x)\right\}$ which contains $b$. Consider $f^{(n+1) k}(x)=$ $f^{n k}\left(f^{k}(x)\right)$. As before, there are three possible types of locations of $f^{(n+1) k}(x)$. If $f^{(n+1) k}(x)$ is located in a component $V$ of $X \backslash\left\{f^{k}(x)\right\}$ which contains neither $x$ nor $b$, then $V$ is inside $B$ and it follows by Lemma 2.5 applied to $V$ and $f^{n k}$ that there is a periodic (actually, $f^{n k}$-fixed) point (in case (2) of the lemma, cutpoint) inside $B$, a contradiction. 
On the other hand, if $f^{(n+1) k}(x)$ is contained in the component of $X \backslash\left\{f^{k}(x)\right\}$ containing $x$, then we can consider $V$ as the component of $X \backslash\left\{x, f^{k}(x)\right\}$ containing $\left(x, f^{k}(x)\right)$. Again, Lemma 2.5 applied to $V$ and $f^{n k}$ implies that there is a periodic (actually, $f^{n k}$-fixed) point (in case (2) of the lemma, cutpoint) inside $B$, a contradiction. Hence $f^{(n+1) k}(x)$ is contained in the component of $X \backslash\left\{f^{k}(x)\right\}$ which contains $b$. By induction we see that for all integers $n \geq 1$ we have that $f^{n k}(x)$ is contained in the component of $X \backslash\left\{f^{k}(x)\right\}$ which contains $b$.

Consider the point $b$. Suppose that $f^{t}(b) \in(d, b)$. Now the arguments from the previous paragraph show that for any $r>1$ the point $f^{r t}(b)$ belongs to the component of $X \backslash\left\{f^{t}(b)\right\}$ containing $d$. Consider $f^{k t}$-images of $b$ and $x$; it follows that $f^{k t}(b)$ is in the component of $X \backslash\{b\}$ containing $d$, while $f^{k t}(x)$ is in the component of $X \backslash\{x\}$ containing $b$. By Lemma 2.5 this implies that there exists a periodic point in $B$ (in case (2) of the lemma, cutpoint), a contradiction.

Now consider a point $y \in(d, b)$. Suppose that for some $t$ we have that $f^{t}(y) \in$ $(d, b)$. Then $f^{t}(y)$ belongs either to the component of $X \backslash\{y\}$ containing $d$ or to the component of $X \backslash\{y\}$ containing $b$. Consider first the case when $f^{t}(y)$ belongs to the component of $X \backslash\{y\}$ containing $d$. Then the component $V$ of $X \backslash\{y\}$ which contains $f^{t}(y)$ must contain $d$. Now the arguments from the previous paragraph show that for any $r>1$ the point $f^{r t}(y)$ belongs to the component of $X \backslash\left\{f^{t}(y)\right\}$ containing $d$. Consider $f^{k t}$-images of $y$ and $x$; it follows that $f^{k t}(y)$ is in the component of $X \backslash\{y\}$ containing $d$, while $f^{k t}(x)$ is in the component of $X \backslash\{x\}$ containing $b$.

Now the argument depends on the mutual location of $y$ and $x$. Suppose that $y$ separates $x$ from $d$ (and so the order of points is $d<y<x<b$ ). Then we are exactly in the situation of Lemma 2.6 as applies to the arc $[y, x]$ and the map $f^{k t}$. Hence there exists a $f^{k t}$-fixed point in $(y, x)$, a contradiction with the properties of $d$. Now assume that $x$ separates $y$ from $d$ (and so the order of points is $d<x<y<b$ ). Then Lemma 2.5 applies to the component $V$ of $X \backslash\{x, y\}$ containing $(x, y)$ and to the map $f^{k t}$ and shows that there is a periodic (actually, $f^{k t}$-fixed) point (in case (2) of the lemma, cutpoint) inside $B$, a contradiction. Since we have considered all possible cases and they all lead to a contradiction, we see that the case when $f^{t}(y)$ belongs to the component of $X \backslash\{y\}$ containing $d$ is impossible. Hence the order of points must be $d<y<f^{t}(y)$. The last claim of the lemma immediately follows.

We are ready to prove Theorem 1.9. Indeed, suppose that $b$ is a recurrent point of arc type which is not a limit point of periodic points. We may assume that there exist a point $d$ and a sequence $\left\{n_{k}\right\}$ such that points $f^{n_{k}}(b)$ belong to $(d, b)$ and converge to $b$, while the component $B$ of $X \backslash\{d, b\}$ containing $(d, b)$ contains no periodic points. Then by Lemma 3.2 we immediately get a contradiction, as by this lemma point $b$ cannot be mapped to $(d, b)$ at all.

To prove Theorem 1.11 we need to work more; in particular we need to take into account the fact that topological polynomials have finitely many critical points whose behavior greatly influences the dynamics of the map (as an example of such influence one can consider Lemma 2.9, which plays a useful role in what follows; in fact, arguments in the proof of the following lemma rely upon Lemma 3.2 and Lemma 2.9). Recall that for brevity we write $f, J, \mathrm{PC}$ instead of $f_{\sim}, J_{\sim}, \mathrm{PC}_{\sim}$ respectively. 
Lemma 3.3. Let $f$ be a topological polynomial with dendritic Julia set $J$ and $X \subset J$ be an invariant dendrite. Suppose that $b$ is a limit point of $x \in X$ of arc type. Then $b$ belongs to the closure $\overline{\mathrm{PC}(X)}$ of the set $\mathrm{PC}(X)$ of all periodic cutpoints of $\left.f\right|_{X}$.

Proof. By way of contradiction we may assume that $b \notin \overline{\mathrm{PC}(X)}$. Since $b$ is a limit point of arc type, there exists an arc $[a, b] \subset X$ and a sequence of images $f^{n_{k}}(x) \in(a, b)$ of $x$ which converge to $b$. On the other hand, the assumption that $b \notin \overline{\mathrm{PC}(X)}$ implies that there exists $d \in(a, b)$ such that the component $B$ of $X \backslash\{d, b\}$, containing $(d, b)$, does not contain periodic cutpoints. By Lemma 3.2 this implies that $b$ never enters $(d, b)$, and for every point $y \in(d, b)$ and any number $m$ such that $f^{m}(y) \in(d, b)$, we have that $f^{m}(y) \in(y, b)$. As before, we introduce the following order among points of $[a, b]: z<y$ means that $y \in(z, b]$. Then by Lemma 3.2 we may assume that $x, f^{n_{1}}(x), \ldots, f^{n_{k}}(x), \ldots$ are all images of $x$ which enter $(d, b)$ and that in fact $x<f^{n_{1}}(x)<f^{n_{2}}(x)<\ldots, f^{n_{i}}(x) \rightarrow b$.

Consider critical points of $f$. Some of them never enter $B$. Let $c_{1}, \ldots, c_{w}$ be all critical points of $f$ which do enter $B$ for the first time under the powers $r_{1}, \ldots, r_{w}$ of $f$, respectively. Observe that since we can choose $d$ arbitrarily close to $b$, we may assume that $r_{i}>0$ for each $i$. However, the proof is also valid if some $r_{i}$ equal zero. Notice also that the points $f^{r_{i}}\left(c_{i}\right)$ do not have to belong to $(d, b)$. It is easy to see that then there exists a point $z \in(d, b)$ such that the following hold:

(1) all points $f^{r_{i}}\left(c_{i}\right), i=1, \ldots, w$, belong to the component of $X \backslash\{z\}$ containing $d$, and

(2) for every $i=1, \ldots, w$ the arc $\left[f^{r_{i}}\left(c_{i}\right), z\right]$ intersects the arc $[d, z]$ over a non-degenerate arc $\left[v_{i}, z\right]$.

Choose a big number $\gamma$ such that $z<f^{n_{\gamma}}(x)$. Construct a set $I$ as follows. Set $q=n_{\gamma+1}-n_{\gamma}$ and consider the union $I$ of all images of $T=\left[f^{n_{\gamma}}(x), f^{n_{\gamma+1}}(x)\right] \subset$ $[x, b]$ under $f^{q}$. Since $f^{q}(T)$ is non-disjoint from $T$, the set $I$ is connected. Clearly, $I$ and its closure map to themselves under $f^{q}$. The same holds for $f$-images of $I$ and $f$-images of its closure $\bar{I}$. Let us show that all critical points $c_{i}, i=1, \ldots, w$, are disjoint from the set $\bar{I}$.

First we claim that images of $T$ never enter the union $Z$ of components of $X \backslash\left\{d, f^{n_{\gamma}}(x)\right\}$ which accumulate upon $f^{n_{\gamma}}(x)$ but are disjoint from $\left(f^{n_{\gamma}}(x), b\right)$ (observe that by the choice of $z$ and $\gamma$ all points $f^{r_{i}}\left(c_{i}\right)$ belong to one of such components, namely to the component which contains $z$ ). Otherwise let $t$ be the least such number where $f^{t}(T)$ enters a component $A \subset Z$. The union $Q$ of all iterated $f^{q}$-images of $f^{t}(T)$ is then a connected set which maps to itself by $f^{q}$. If $Q$ is contained in $\bar{B}$, then by Theorem 2.7 there are infinitely many periodic cutpoints in $\bar{Q} \subset \bar{B}$, which implies that there are infinitely many periodic cutpoints in $B$, a contradiction.

On the other hand, by Lemma 3.2. $d \notin Q$. Hence we see that $\bar{Q}$ must "get out of $B$ through $b$ ", i.e., that $Q$ must contain $b$. As $Q$ is connected and contains points of $A$, we see that $f^{n_{\gamma}}(x) \in Q$. This means that for some $i$ we have $f^{n_{\gamma}}(x) \in$ $f^{t+q i}(T)$. Hence there is a point $y \in T$ such that $f^{t+q i}(y)=f^{n_{\gamma}}(x)$. Since $B$ contains no periodic cutpoints, $y \neq f^{n_{\gamma}}(x)$. On the other hand, by Lemma 3.2, $y \neq f^{n_{\gamma}}(x)$ is also impossible. This contradiction shows that $f$-images of $T$ never enter components of $X \backslash\left\{d, f^{n_{\gamma}}(x)\right\}$ which accumulate upon $f^{n_{\gamma}}(x)$ but are disjoint from $\left(f^{n_{\gamma}}(x), b\right)$. In particular, $f^{n_{\gamma}}(x)$ is an endpoint of $\bar{I}$ and, by continuity, all $f$-images of $\bar{I}$ are disjoint from $Z$. 
Clearly, the set $Y=\bigcup_{i=0}^{q-1} \bar{I}$ is $f$-invariant. Consider a component $Y^{\prime}$ of $Y$ such that $\bar{I} \subset Y^{\prime}$. Then by the above the set $Y^{\prime}$ is disjoint from $Z$ and $f^{n_{\gamma}}(x)$ is an endpoint of $Y^{\prime}$. Since $f^{q}$ maps $\bar{I}$ to itself, there exists the smallest $s$ such that $f^{s}\left(Y^{\prime}\right) \subset Y^{\prime}$, while sets $Y^{\prime}, f\left(Y^{\prime}\right), \ldots, f^{s-1}\left(Y^{\prime}\right)$ are pairwise disjoint. Let us show that all sets $f^{i}\left(Y^{\prime}\right), 1 \leq i \leq s-1$, are disjoint from $B$. Indeed, since $f^{n_{i}}(x) \rightarrow b$ while $f^{n_{i}}(x) \in(d, b)$, then $b \in Y^{\prime}$. This implies that if $f^{i}\left(Y^{\prime}\right)$ is non-disjoint from $B$, then $f^{i}\left(Y^{\prime}\right) \subset \bar{B}$ (recall that by the preceding paragraph this component is disjoint from $Z$, while on the other hand the point $b$ does not belong to $f^{i}\left(Y^{\prime}\right)$, and so $f^{i}\left(Y^{\prime}\right)$ cannot exit $B$ through $\left.b\right)$. Since $f^{i}\left(Y^{\prime}\right)$ maps to itself by $f^{s}$, we see by Lemma 2.5 that there are periodic cutpoints in $B$, a contradiction. Hence all sets $f^{i}\left(Y^{\prime}\right), 1 \leq i \leq s-1$, are disjoint from $B$.

Now, since points $f^{r_{i}}\left(c_{i}\right)$ belong to $Z$ for all $i=1, \ldots, w$, it follows that points $c_{i}, i=1, \ldots, w$, do not belong to $Y$. Therefore, by the preceding paragraph and by the choice of critical points $c_{i}$ we see that the critical points of $f^{s}$ which belong to $Y^{\prime}$ never enter $B$ under iterations of $f$. Hence the minimal $f^{s}$-invariant continuum $T$ containing all critical points of $f^{s}$ which belong to $Y^{\prime}$ is disjoint from $B$. On the other hand, by Lemma 2.9 every cutpoint of $\bar{I}$ eventually maps to $T$ and then stays in $T$ under iterations of $f^{s}$ while staying away from $B$ under other iterations of $f$ (because under other iterations of $f$ this cutpoint will stay away from $B$ as shown above). Applying this to the point $f^{n_{\gamma+1}}(x)$ we see that its forward orbit cannot converge to $b$ from within the arc $\left[f^{n_{\gamma+1}}(x), b\right]$, a contradiction with our assumptions, which completes the proof.

Recall that in Theorem 1.11 we deal with sets of all limit points, not only limit points of arc type. To study this more general situation we use laminations. We investigate what corresponds to limit points of arc type (and non-separating type) in the language of laminations. Let $x$ be a persistent cutpoint; then we may assume that $G_{x}$ and all its images are gaps or leaf-classes and that the bases of all images of $G_{x}$ consist of the same finite number of points. If $b$ is a limit point of arc type of $x$, then it follows that a sequence of images of $G_{x}$ converges onto an edge $\ell$ of $G_{b}$ or onto a bud $G_{b}$ (which in this case we assume to be a degenerate $\ell$ ) so that each next image in this sequence of images of $G_{x}$ separates the previous images in this sequence from $G_{b}$ (in such cases we say that a sequence of images of $G_{x}$ converges onto $\ell$ from one side). On the other hand, in the case of a limit point $b$ of non-separating type, any sequence of images of $G_{x}$ which converges to $G_{b}$ converges either to an endpoint of $\ell$ or to a bud $G_{b}$ (thus, diameters of these images of $G_{x}$ converge to zero), and we may assume that a converging sequence of images of some $G_{x}$ with separation properties, constructed in the definition of convergence of arc type, does not exist for $b$ (or for $G_{b}$ ).

Thus, these two types of limits can be distinguished in the disk as well. By $\omega^{a}\left(G_{x}\right)$ we mean the set of all leaves and buds approached by images of $G_{x}$ from one side and by $\omega^{n s}\left(G_{x}\right)$ the set of all the other limits of $G_{x}$. Then $p_{\sim}$ maps $\omega^{a}\left(G_{x}\right)$ to $\omega^{a}(x)$ and $\omega^{n s}\left(G_{x}\right)$ to $\omega^{n s}(x)$.

Lemma 3.4. The set $\omega^{a}\left(G_{x}\right)$ is $\sigma_{d}$-invariant and dense in $\omega\left(G_{x}\right)$. The set $\omega^{a}(x)$ is $f$-invariant and dense in $\omega(x)$.

Proof. Let $b$ be a limit point of $x$ of arc type. Then, by definition, there is an edge $\ell$ of $G_{b}$ onto which a sequence of images of $G_{x}$ converges from one side (in the degenerate case when $G_{b}$ is a bud, we consider it to be equal to $\ell$ ). Clearly, the 
same can then be said about the image $\sigma_{d}\left(G_{b}\right)$ of $G_{b}$. Thus, $\omega^{a}\left(G_{x}\right)$ is $\sigma_{d}$-invariant and $\omega^{a}(x)$ is $f$-invariant as desired.

Now, suppose that $\omega^{a}(x)$ is not dense in $\omega(x)$. Then it is easy to see that there exist arcs $I \subset K \subset \mathbb{S}^{1}$ such that the following hold:

(1) infinitely many images of $G_{x}$ intersect $I$, while the diameters of all such images of $G_{x}$ converge to zero so that from some time on all images of $G_{x}$ which intersect $I$ have bases contained in $K$ (i.e., there exists $k$ such that for all $m \geq k$ with $\sigma_{d}^{m}\left(G_{x}\right)$ non-disjoint from $I$ we will have that the basis of $\sigma_{d}^{m}\left(G_{x}\right)$ is contained in $\left.K\right)$, and

(2) the sets $\omega^{a}\left(G_{x}\right)$ and $K$ are disjoint.

Suppose that $\sigma_{d}^{n}\left(G_{x}\right)$ has a very small diameter and is non-disjoint from $I$. Since $\sigma_{d}$ is expanding, it follows that for some $t<n$ the set $\sigma_{d}^{t}\left(G_{x}\right)$ is very close to a critical leaf $c$ and that $\sigma_{d}^{n-t}(c) \in K$. As there are finitely many critical leaves, it follows that there is a critical leaf $c$ which is a one-sided limit of images of $G_{x}$ (thus, $\left.c \in \omega^{a}\left(G_{x}\right)\right)$ and which enters $K$. Clearly, this is a contradiction.

Theorem 1.11 immediately follows from Lemma 3.3 and Lemma 3.4 Moreover, we are ready to prove Corollary 1.12, Indeed, suppose that $f: J \rightarrow J$ is a topological polynomial with dendritic Julia set $J$ and $\mu$ is an $f$-invariant probability measure. Clearly, any non-periodic point has zero $\mu$-measure. In particular, this holds for critical points which map to endpoints of $J$. It follows that $\mu$ can be represented as a convex combination of two invariant probability measures, $\mu_{e}$ (supported on the set of all endpoints of $J_{\sim}$ ) and $\mu_{c}$ (supported on the set of all cutpoints of $J_{\sim}$ ). Now, by the Poincaré Recurrence Theorem Poi1890 the set of recurrent persistent cutpoints has full $\mu_{c}$-measure. On the other hand, by Theorem 1.11 all recurrent persistent cutpoints belong to the closure of the set of periodic cutpoints. This completes the proof of Corollary 1.12 .

\section{ACKNOWLEDGMENTS}

The author would like to thank L. Oversteegen, R. Ptacek and V. Timorin for useful discussions. He is also grateful to the referee for useful and thoughtful comments.

\section{REFERENCES}

[AEO07] G. Acosta, P. Eslami, L. Oversteegen, On open maps between dendrites, Houston Journal of Mathematics 33 (2007), 753-770. MR2335734 (2008k:54014)

[ALM00] L. Alseda, J. Llibre, M. Misiurewicz, Combinatorial Dynamics and Entropy in Dimension One, Adv. Ser. in Nonlinear Dynamics 5, World Scientific, Singapore (2000). MR1807264 (2001j:37073)

[Blo95a] A. Blokh, Rotation Numbers, Twists and a Sharkovskii-Misiurewicz-type Ordering for Patterns on the Interval, Ergodic Theory and Dynamical Systems 15 (1995), 1-14. MR1314966 (96c:58058)

[Blo95] A. Blokh, The Spectral Decomposition for One-Dimensional Maps, Dynamics Reported 4 (1995), 1-59. MR1346496 (96e:58087)

[Blo80s] A. Blokh, On Dynamical Systems on One-Dimensional Branched Manifolds. I, II, III (in Russian), Theory of Functions, Functional Analysis and Applications, Kharkov, 46 (1986), 8-18; 47 (1986), 67-77; 48 (1987), 32-46. MR.0865783 (88j:58053) MR0916445(89i:58056), MR0916457(89i:58057)

[BFMOT10] A. Blokh, R. Fokkink, J. Mayer, L. Oversteegen, E. Tymchatyn, Fixed point theorems for plane continua with applications, preprint arXiv:1004.0214, 107 pages (2010), to appear in Memoirs of the Amer. Math. Soc. 
[BL02a] A. Blokh, G. Levin, An inequality for laminations, Julia sets and "growing trees", Ergod. Th. and Dynam. Sys., 22 (2002), 63-97. MR.1889565 (2003i:37045)

[BM97] A. Blokh, M. Misiurewicz, New Order for Periodic Orbits of Interval Maps, Ergodic Theory and Dynamical Systems 17 (1997), 565-574. MR1452180 (98i:58191)

[BOPT11] A. Blokh, L. Oversteegen, R. Ptacek, V. Timorin, Dynamical cores of topological polynomials, to appear in Proceedings of the Conference "Frontiers in Complex Dynamics (celebrating John Milnor's 80th birthday)", arXiv:1106.5022.

[MT89] P. Minc, W. Transue, Sharkovskii's theorem for hereditarily decomposable chainable continua, Trans. Amer. Math. Soc. 315 (1989), 173-188. MR.965302 (89m:54054)

[Nit80] Z. Nitecki, Periodic and limit orbits and the depth of the center for piecewisemonotone interval maps, Proc. Amer. Math. Soc. 80 (1980), 511-514. MR581016 (81j:58068)

[Poi1890] H. Poincaré, Sur le problème des trois corps et les équations de la dynamique, Acta Math. 13 (1890), 1-270.

[Sha64] A. N. Sharkovskiy, Co-existence of the cycles of a continuous mapping of the line into itself, Ukrain. Mat. Zh. 16 (1964), 61-71. MR.0159905 (28:3121)

[Sha64a] A. N. Sharkovskiy, Non-wandering points and the center of a continuous map of the line into itself (in Ukrainian), Dop. Acad. Nauk Ukr. RSR, Ser. A (1964), 865-868.

[Sha66] A. N. Sharkovskiy, The behavior of a map in a neighborhood of an attracting set (in Russian), Ukr. Math. J., 18 (1966), 60-83. MR0212784 (35:3649)

[Sha66a] A. N. Sharkovskiy, The partially ordered system of attracting sets, Soviet Math. Dokl., 7 (1966), 1384-1386.

[Sha67] A. N. Sharkovskiy, On a theorem of G. D. Birkhoff (in Russian), Dop. Acad. Nauk Ukr. RSR, Ser. A (1967), 429-432.

[Sha68] A. N. Sharkovskiy, Attracting sets containing no cycles (in Russian), Ukr. Math. J. 20 (1968), 136-142. MR0225314(37:908)

[Thu85] W. Thurston, On the geometry and dynamics of iterated rational maps, in: "Complex Dynamics: Families and Friends", ed. by D. Schleicher, A K Peters (2009), pp. 3-110. MR2508255(2010m:37076)

Department of Mathematics, University of Alabama at Birmingham, Birmingham, Alabama 35294-1170

E-mail address: ablokh@math.uab.edu 